\title{
The Cell and its Worry about the Water
}

\section{Martin Vlček}

Non-profit research group, Prague, Czech Republic

*Corresponding author: Martin Vlček, eFunctionality, non-profit research group, Prague, Czech Republic, Tel: 727164850; E-mail: martin.vlcek@efunctionality.eu Rec date: May 14, 2015; Acc date: May 28, 2015; Pub date: May 30, 2015

Copyright: (C) 2015 Vlček M, et al. This is an open-access article distributed under the terms of the Creative Commons Attribution License, which permits unrestricted use, distribution, and reproduction in any medium, provided the original author and source are credited.

\section{Abstract}

The regeneration of elements of water is the basic goal of the control of the cell. These elements are molecules and complex of molecules of different kinds, but at the end they are molecules of water. The control systems try to allow to these elements to optimize their state depending on circumstances, perhaps in advance.
\end{abstract}

The first of these systems is the cytoplasmic membrane together with the apparatus of input mechanisms. It controls the materialistic and non-materialistic input for the regeneration and at the same time it is the source of the primary information about its state and development at time, too.

The other member of the control is the control of the distribution of the energy. This is the control of the ATP economy in connection with the proton economy. The control of the flow of energy is a mean for the regulation of the process of the regeneration on the overall level. It stems out from the overall satisfaction of molecules of water, what is a function of the dynamics of the hydrogen part of molecule. The localizations are mitochondria and occasionally plastids.

The system named EMO controls the procedure of the regeneration on the basis of information concerning the successfulness of elements involved in it. The system EM1 controls the dynamics of the boundary of the cell on the basis of information about the tension on it. Both systems converge into the area of the nucleus.

The optimization of the existence of the cell itself as whole, besides the regeneration, is the second goal of the control of the cell. New global criteria arise for this sake and are used during the control.

\section{Introduction}

\section{Analogy}

The basic properties of every object are derived from the functioning of its control systems. These systems function on different levels with the same logic, even if they are working with different elements. However, goals and means are the same $[1,2]$.

The submitted work also arises from this logic. It uses findings from analogical systems proved by extended calculations $[3,4]$ and finds and applies them in the cell.

On the other hand, the author realizes that, similarly as with the mentioned objects, it is necessary to prove them experimentally in the cell, too.

\section{Water}

The submitted work stems from the fact that molecules of water are the elements of the system. These are not different sophisticated and more or less complex structures, e.g. proteins. These are only means for the functioning of the cell, for the involvement of the water into the process of regeneration. The basic paradigm of the functioning and thus of the control of the cell stems from it. It stems from the monitoring of the successfulness, occasionally of the satisfaction of elements, it means of molecules of water. This monitoring is made through working and aggregating means, though.
The submitted work stems from the variability of water in the sense of dynamics of the proton and surely of others, still unaware events on the boundary of the molecule with its environment, too. The unknown information about the satisfaction is located in this variability, too. From the analogy with other objects it has to be a function of interactions with the environment. The decisive is their level and the stability. In the case of incoming interactions the speech is about the satisfaction of the molecule, in the case of outgoing ones about its successfulness. These are the criteria used in the process of the control.

\section{Mitochondria}

Mitochondria play in the system probably a quite basic role in the existence of the water. There happens to the interconnection of the energetic systems of the cell, staying on ATP, and of the water, staying on the dynamics of the hydrogen in a broader sense.

The interconnection happens on two levels. Firstly, the regeneration proceeds on the level of the cell into the cytoplasm and means mainly the materialistic reactions on events. This is the establishing of prepositions for the regeneration of the water. Secondly, it goes about the redistribution of a part of energy into molecules of water themselves and the facilitation of a real, means inner regeneration itself. 
Page 2 of 4

\section{The Control of the Cell}

\section{The Central Axe - the Regeneration}

The basic function of the cell, its central axe, is, besides the control of the existence of the cell as whole, the regeneration of molecules of water. It consists not only into the solution of sequels of their activities or changes into the environment, but also into a preparation to them, occasionally into an avoidance of unwanted states.

A molecule of water enters into a variety of interactions and structures thanks to its week bonds mainly by hydrogen bridges. The distribution of hydrogen, not only in the form of bridges, around the oxygen, represents an inexhaustible variety of possibilities. But the most substantial is the fact that the molecule itself prefers to some states over others. These states are said to be more stable. For the satisfaction of the water is also important into what bonds with what molecules it enters. In addition, some dynamics of states serves as a communicative mean. Thus it is possible to say, that the world of interconnections of a molecule of water is very diverse and very dynamic and that it is subjected to the process of the regeneration into the cell.

On the beginning of the system of the regeneration of the cell stays the membrane of the cell as a provider of materialistic, but also nonmaterialistic inputs. It is also a source of basic impulses for the following control. These impulses spread toward the nucleus of the cell, their aggregation and projection over the activity of the nucleus happens. By return, the state of the membrane is influenced.

Around the central axe and its energetic control there are two other control systems. Both work with the satisfaction of the successfulness of molecules of water. The successfulness of individual molecule of water into the process of the regeneration is processed by the system named EM0. A system named as EM1 solves the successfulness of molecules of water on the boundary and monitors it through complexes into the cytoplasmic membrane.

\section{The Input System}

The input system represents a relatively independent and highly important element in the regulative structure of the cell. This is a complex of the cytoplasmic membrane with all the apparatus of more or less anchored protein receptors, channels and agents.

Besides the function connected with the input of necessary materials it is the place of the arising of primary information about prepositions of the regeneration, means about disposable inputs in the environment. Impulses from it go perhaps through fibrillary structures and occasionally through water structures into the area of the nucleus and form a gradient in what other higher control systems work.

So, there is a bifurcation of ways into the input system. The materialistic part proceeds through the cytoplasmic more or less controlled pathway to places of final activities. The signal part inputs into informatics structures and brings information about the state in individual parts of the input boundary.

Thus the input, primary boundary is the input gate of the cell and is controlled mainly from the area of the nucleus by a structure named EM1.

\section{TOT}

\section{The Control of the Axe - TOT}

The lowest level of the control is connected with the production and the distribution of energy. Its particularity is among others in the fact that it doesn't stem from the feedback of individual elements making regeneration, but from the overall information about the state of the regeneration. This control system is named TOT.

The substance of TOT is in the control of the activity of mitochondria and occasionally of plastids in the production and distribution of energy, in cells and partly in molecules of water.

The role of TOT is to optimize the process of the regeneration, mainly from the energetic point of view. It means to stem from the presence of certain indicators of the state of the regeneration in the cytoplasm and to react in a manner to shift the overall regeneration forward. It is possible that TOT reacts besides chemical and physical reagents on their dynamics, too, where the volatility of their concentration is a demonstration of a certain state of the regeneration.

TOT by its activity interconnects the energetic systems of the cell and of the molecule of the water, the system of ATP and the system of $\mathrm{H}+$. After that it influences the distribution into consumption and reserves, in the cell and in the water. Through the way of the energetic control the overall flow of the regeneration is managed.

\section{EM0}

\section{Regeneration}

The task of EM0 is to control structures involved in the regeneration. It doesn't evaluate the successfulness of the overall regeneration, its final effect. TOT does it, but EM0 on the basis of the successfulness of individual participating elements, mainly of proteinous elements of the regeneration, optimizes the process of the regeneration itself.

Perhaps at this place it is possible to note, that the protein forms mainly a complex for a water chemical reaction. There is an involvement of a molecule of water in this complex into the regeneration and thus the successfulness, evaluated finally on the basis of the successfulness of the complex is the successfulness of molecules of water, of elements of the cell, too.

\section{Information}

In the contrast with TOT, where the feedback happens on the basis of the overall indicators of the regeneration, e.g. of the concentration of some substances in the cytoplasm, here the origin of the control is the information coming from complexes of elements. It is probable, that this information is built on an indicator of the consumption of energy, means of the involvement into regeneration. E.g. on the local concentration of nearby products in the fissure of ATP.

It is important, that the relevant information is not only the concentration of these products in a given locality, means in a given step of the regeneration, but their volatility at time, too. Then EM0 intervenes into areas not only with a high consumption of energy, but mainly with a volatile one. 


\section{Aggregation}

It is not possible to understand the activity of the nucleus as a sum of activities just coming in activators, what will only mechanically "play back their song", means will transcribe appropriate gene and "the long day will continue by its rate" (Wodehouse). The activity of the nucleus underlies to a "higher interest", to the interest of the cell. The cell have to evaluate what is the actual state of the regeneration and regarding to the state of the environment, means e.g. to these activators, too, what is the correct procedure.

The result of the aggregation of the information is the establishment of the overall orientation of the necessary reaction, procedure in the regeneration. It is probable, that it is not only a mechanical solution of the actual state by a set of chemical reactions, but a finding of an optimal strategy. This strategy starts to be gradually realized, by a way of a desegregation it is transcribed into a local level and throughout a way of the feedback about the successfulness eventually modified. It can go nevertheless about the strategy, means a step, totally not resulting from a chemical folder, but thanks to a "memory" of EM0 it e.g. prevents to some undesirable state.

\section{Reconstruction}

The information spreads and aggregates into the cytoplasm apparently into the area of the nucleus through a way of fibril structures or structures of water in a form of dynamic events. There happens a total aggregation of this information in the area of the nucleus and a turning round of the flow of the control. Thus in one direction spreads and is aggregated the information about the successfulness, in an opposite direction spreads and is desegregated the information about the flow of newly synthesized proteins and thus about the flow of energy, too.

In the process of the optimization of the regeneration there are two aspects of the control. In the first one it is necessary to divide the overall potential of the proteosynthesis in a manner responding to the overall necessity of the cell. It is necessary to find out the most exposed areas of the regeneration and to intervene there. Inversely, it is possible to damp areas less active. So it is a quantitative control. The mechanism of the control of the local activity itself apparently consists in a modification of the distribution of proteins to their places of destination.

In the second aspect it goes around a qualitative control, when structures of ways of the distribution of proteins are changed depending on the activity of appropriate areas. Successful areas and ways attract other expansion of ways of the distribution.

\section{EM1}

\section{The Role of EM1}

The task of EM1 is to control events on the boundary of the cell with the environment. It concerns both materialistic and nonmaterialistic events.

The impulse for its activity is the tension, what arises on the boundaries in the case, when near positions are submitted to a different influence of the environment. The substance of the activity of EM1 is to do a reconstruction of the boundary what will cancel this tension.

\section{Principle}

The principle is to some extent similar as at EM0. The basic difference is in the fact, that it follows the successfulness of complexes not in the cytoplasm but in the cytoplasmic membrane, on the boundary. The intervention of the system goes not into places with highest successfulness, but into places with a maximal tension. Similarly as with EM0 an important role plays its volatility.

An information concerning the tension spreads in EM1 toward the centre, means toward the nucleus, until its aggregation and a finding out of the maximal tension happens. The activity of the cell is thus cast down into places, where it is the most necessary. The reaction of the cell is not a collective reaction, where every impulse activates its way and it is expected that all will terminate well.

An impulse to the reconstruction spreads from the centre and its sequel is the suppression of the maximal or the most important tension, or complex of tensions. In sum it is possible to describe this control for new similarly as at EM0, means with two aspects quantitative and qualitative control.

\section{Satisfaction and Successfulness}

The tension on the boundary of the cell is formed through more or less complex structure of proteins, contributing through materialistic or non-materialistic inputs. There is some interest for these inputs in the cell and this is manifested on the overall evaluation of a given input as been more or less successful. The similarity of these inputs is compared with the similarity of their successfulness. And if some similar inputs are evaluated as non-similarly successful, EM1 evaluates it as a tension on the boundary. This is the impulse been solved afterwards.

But it is also a quantity, on what basis a new, global quantity arises and this is the satisfaction of the overall cell. It is proportional to the overall average successfulness of inputs, but inversely proportional to its volatility. This quantity is the value what orients the global behavior of the cell.

\section{Consciousness}

The maximal tension, more exactly the tension what succeeds to arrive till the final level in the area of the nucleus, is possible to name as a consciousness. The more exact name than the maximal tension is the tension describing the most important event, tension on the boundary. And the most important means that it has to be preferably solved.

\section{The Holistic Principle}

The boundary of the cell is a place where a new object arises as a new quality. By the fact that only one consciousness arises, only one aggregated and evaluated tension been solved and thus influencing the following behavior of the cell, the new quality arises. It is already not a sum of individualities been more or less in contradiction; it is the only and unique strategy. And this consciousness can solve not only the actual tension of the border but perhaps the tension been only coming in the future.

Besides the global satisfaction what determines the gradient for the movement of the cell, the global tension - consciousness gives an impulse allowing to the cell to orient itself throughout this movement, means to find out solutions. 
The third global quantity is the result of the aggregation in EM0. It is a quantity showing the global success of the regeneration, evaluated not on the basis of e.g. cytoplasmic concentration but on the basis of information coming from individual elements, in a final instance from the water. Only this quantity allows a goal-directed control of the process of the regeneration and to fill in the first mission of the cell the care for the water.

By a mutual cooperation of these three quantities the behavior of the cell is control. It seems, that dominant are quantities connected with EM1 and that the quantity connected with EM0 serves to the choice of strategies from the point of view of the energetic demand.

\section{Conclusion}

The philosophy of the functioning of the cell, means of its control structures, stems upon two points of view. The first one solves the satisfaction of elements, the second one the satisfaction of the new object built upon these elements.

The first one solves the regeneration of molecules of water, and thus the process of satisfying of building elements. It reaches it throughout a control depending on different criteria. Firstly it does it by a supply of materialistic components of the regeneration. Afterward controls its energetic flow throughout global criteria of the successfulness of the regeneration- TOT. Afterward controls the regeneration from the point of view of the successfulness of its executors EM0.
The second one solves the tension of the overall border - EM1, so it returns to the beginning.

And just on the overall boundary, been indispensable for the existence of the cell, a new quality arises. An already new strategy connected with the new object - the cell, is implemented by a controlled input into the consciousness and occasionally by a generation of proper tensions.

\section{References}

1. Vlcek M (2008) Functioning of the Object. unpublished material.

2. Vlcek M (2015) Firm without Subordinations. in American Journal of Economics 5: 3.

3. Vlcek M (2010) Movers of the Economy - Model of Interactions, in Developments in Economic Theory and Policy proceedings, Bilbao, Spain.

4. Vlcek M (2014) New Object Discovered through the Stock Market", in International Conference on Economics and Finance Research proceedings, Seoul, South Korea.
This article was originally published in a special issue, entitled:

"Cytopathology", Edited by Borislav A. Alexiev, University of Maryland Medical Center, USA 